\title{
A Feature Ranking Strategy to Facilitate Multivariate Signal Classification
}

Lalit Gupta $^{1}$, Srinivas Kota ${ }^{1}$, Swetha Murali ${ }^{1}$, Dennis Molfese $^{2}$, \& Ravi Vaidyanathan ${ }^{3}$

${ }^{1}$ Department of Electrical \& Computer Engineering, Southern Illinois University

Carbondale, IL 62901, U.S.A.

${ }^{2}$ Developmental Neuropsychology Laboratory at the Birth Defects Center, University of Louisville, Louisville, KY 40292, U.S.A.

${ }^{3}$ Department of Mechanical Engineering, University of Bristol Bristol BS8 1TR, U.K. 


\begin{abstract}
A strategy is introduced to rank and select principal component transform (PCT) and discrete transform (DCT) transform coefficient features to overcome the curse of dimensionality frequently encountered in implementing multivariate signal classifiers due to small sample sizes. The criteria considered for ranking include the magnitude, variance, inter-class separation, and classification accuracies of the individual features. The feature ranking and selection strategy is applied to overcome the dimensionality problem which often plagues the implementation and evaluation of practical Gaussian signal classifiers. The applications of the resulting PCT- and DCT-Gaussian signal classification strategies are demonstrated by classifying single-channel tongue-movement ear-pressure signals and multi-channel event-related potentials. Through these experiments, it is shown that the dimension of the feature space can be decreased quite significantly by means of the feature ranking and selection strategy. The ranking strategy not only facilitates overcoming the dimensionality curse for multivariate classifier implementation but also provides a means to further select, out of a rank-ordered set, a smaller set of features that give the best classification accuracies. Results show that the PCT- and DCT-Gaussian classifiers yield higher classification accuracies than those reported in previous classification studies on the same signal sets. Amongst the combinations of the two transforms and four feature selection criteria, the PCT-Gaussian classifiers using the maximum magnitude and maximum variance selection criteria gave the best classification accuracies across the two sets of classification experiments. Most noteworthy is the fact that the multivariate Gaussian signal classifiers developed in this paper can be implemented without having to collect a prohibitively large number of training signals simply to satisfy the dimensionality conditions. Consequently, the classification strategies can be beneficial for designing personalized human-machine-interface (HMI) signal classifiers for individuals from whom only a limited number of training signals can reliably be collected due to severe disabilities.
\end{abstract}

Key Words: Curse of dimensionality, Discrete cosine transform, Ear-pressure signal classification, Event related potential classification, Feature ranking, HMI, Multivariate signal classification, Principal component transform. 


\section{INTRODUCTION}

In signal classification problems, the dimension of the input signal space depends on the sampling rate, the duration over which the signal is sampled, and the number of recording channels. It is not unusual for the resulting dimension to be high especially for signals that are voluntarily generated by humans for human-machine-interface (HMI) control and communication applications. Examples of such signals include speech [1]-[3], ear pressure signals [3],[4], electromyographic signals [5],[6], electroencephalogram (EEG) and event-related potential (ERP) brainwaveforms[6]-[9], and gestures [10],[11]. Due to practical issues related to the data acquisition methods, lack of concentration, discomfort, and fatigue, it may not always be possible to collect enough reliable signals to exceed the dimension of the signal space. Consequently, the signal clusters are sparsely represented in the signal space and the estimates of the classifier parameters are either inaccurate or cannot be computed. One of the most viable options for overcoming this "curse of dimensionality" is to decrease the dimension of the signal space, typically, through feature selection. We focus on features generated by the principal component transform (PCT) and the discrete cosine transform (DCT). The transforms do not directly decrease the dimension because the transformed feature space has the same dimension as the input signal space. The PCT and DCT, however, efficiently pack information into a small number of transform coefficients. Consequently, dimensionality reduction in the transformed feature space can be achieved by discarding the coefficients that carry the least useful information.

In this paper, we introduce a feature selection strategy based on the rankings of the features. The criteria considered for ranking include the magnitude, variance, inter-class separation, and classification accuracies of the individual features. Because the feature vectors are frequently assumed to be Gaussian, we focus on overcoming the dimensionality-related problem in the implementation of Gaussian signal classifiers using the PCT/DCT feature ranking and selection strategy. The application and evaluation of the resulting PCT- and DCT-Gaussian signal classification strategies are demonstrated by classifying single-channel ear-pressure signals of eight subjects and multi-channel event-related potential (ERP) brain signals of three subjects. These particular signal classification problems are selected because they are typical of signal classification problems in which the curse of dimensionality is known to occur frequently. Furthermore, the signals are also typical of signals that can be used to control HMIs [3], [4], [11]. 


\section{FEATURE RANKING AND SELECTION}

Feature generation using transforms such as the PCT and DCT can be expressed, in general, as

$$
\tilde{Z}=\Phi_{D} Z
$$

where, $\Phi_{D}$ is the $(D \times D)$ transformation matrix and $\tilde{Z}$ is the $D$-dimensional transformed feature vector whose elements are linear combinations of the elements of the $D$-dimensional input signal space vector $Z$. The rows of $\Phi_{D}$ are the basis vectors of the transform. The goal of the feature ranking and selection strategy introduced in this section is to decrease the dimension of the transformed feature space to a specified dimension $d, d<D$, by selecting $d$ rows of $\Phi_{D}$. The $d$ rows are selected as the basis vectors that generate the $d$ highest ranked features according to a specified criterion. Then, the dimensionality reduced feature vector is given by

$$
\hat{Z}=\hat{\Phi}_{d} Z
$$

where $\hat{\Phi}_{d}$ is the $(d \times D)$ dimensionality reduction matrix consisting of the $d$ selected basis vectors.

The ranking of features for classification problems, however, is not straight-forward because classification involves multiple signal classes and multiple feature vectors in each signal class. The features selected according to a given criterion are very likely to differ within each signal class (intraclass differences) and also differ across the classes (inter-class differences). The ranking strategy must yield a common ranking of the features across all signal classes. In order to accomplish this, a generalized strategy consisting of intra-class ranking followed by inter-class ranking is introduced next.

\section{Feature Ranking Strategy}

We will use the following notations to facilitate the description of the ranking strategy:

$C=$ number of signal classes

$N_{c}=$ number of training vectors in class $c, c=1,2, \ldots, C$

$\tilde{Z}(k)=$ feature $k, k=1,2, \ldots, D$, in transformed feature vector $\tilde{Z}$

$\tilde{Z}_{j c}=$ feature vector $j, j=1,2, \ldots, N_{c}$, in class $c$

$\tilde{Z}_{j c}(k)=$ feature $k$ in feature vector $\tilde{Z}_{j c}$

Let

$$
r_{j c}(k)=\operatorname{Rank}\left\{\tilde{z}_{j c}(k)\right\}, \quad k=1,2, \ldots, D .
$$

be a ranking of a feature $\tilde{z}_{j c}(k)$ within each feature vector $\tilde{Z}_{j c}$. The rank $r_{j c}(k)$ for each $j$ may be different within a class $c$. In order to accommodate the different rankings, the intra-class rank of each 
feature within a class $c$ is determined by first summing the ranks of each feature across the $N_{c}$ training vectors of class $c$ according to

$$
s_{c}(k)=\sum_{j=1}^{N_{c}} r_{j c}(k), \quad k=1,2, \ldots, D .
$$

The intra-class rank $q_{c}(k)$ of the feature $\tilde{z}(k)$ is then given by the rank of the rank-sum $s_{c}(k)$. That is,

$$
q_{c}(k)=\operatorname{Rank}\left\{s_{c}(k)\right\}, \quad k=1,2, \ldots, D .
$$

The rank $q_{c}(k)$ may differ across the $C$ signal classes. In order to take these differences into account, the rank of each feature across all the $C$ classes is summed using

$$
s(k)=\sum_{c=1}^{C} q_{c}(k), \quad k=1,2, \ldots, D .
$$

Finally, the ranking of feature $\tilde{z}(k)$ across all $C$ classes is determined from the rank of the rank-sum $s(k)$. That is, the final inter-class ranking $r(k)$ of feature $\tilde{z}(k)$ is given by

$$
r(k)=\operatorname{Rank}\{s(k)\}, \quad k=1,2, \ldots, D .
$$

The steps of the entire ranking strategy can be summarized as

$$
\text { Inter-class } \operatorname{Rank}\left\{\operatorname{Sum}\left\{\operatorname{Intra-class} \operatorname{Rank}\left\{\operatorname{Sum}\left\{\operatorname{Rank}\left\{\tilde{z}_{j c}(k)\right\}\right\}\right\}\right\}\right\}
$$

Based on the ranking, the features with the $d$ highest ranks can be selected to satisfy the dimensionality condition in order to facilitate multivariate classifier design. We assume throughout that the highest ranked feature is ranked 1 and the lowest is ranked $D$. Therefore, the feature with the smallest value in $r(k)$ is the best inter-class feature and the one with the largest value in $r(k)$ is the worst interclass feature. The criteria, which are described next, for determining the rankings can be based on the magnitudes, variances, inter-class separations, and classification accuracies of the features.

\section{Magnitude (MAG) Criterion}

The ranking $r_{j c}(k), k=1,2, \ldots, D$, can be based on the magnitude $\left|\tilde{z}_{j c}(k)\right|$ of $\tilde{z}_{j c}(k)$. That is,

$$
r_{j c}(k)=\operatorname{Rank}\left|\tilde{z}_{j c}(k)\right|, \quad k=1,2, \ldots, D
$$

is a ranking such that the feature with the largest magnitude is assigned rank 1 and the lowest magnitude is assigned $D$. Then, $s_{c}(k), q_{c}(k), s(k)$, and $r(k)$ are given by Equations (4), (5), (6), and (7), respectively. 


\section{Variance (VAR) Criterion}

The intra-class ranking $q_{c}(k)$ can be based on the variance $\operatorname{VAR}\left[\tilde{z}_{c}(k)\right]$ of $\tilde{z}(k)$ in each class $c$. For this case,

$$
q_{c}(k)=\operatorname{Rank}\left\{\operatorname{VAR}\left[\tilde{z}_{c}(k)\right]\right\}, \quad k=1,2, \ldots, D
$$

is a ranking such that the feature with the largest variance is assigned rank 1 and the smallest variance is assigned $D$. Then, $s(k)$, and $r(k)$ are given by Equations (6) and (7), respectively.

\section{Interclass Separation (I-S) Criterion}

The inter-class separation, which is a normalized measure of the separation between 2 cluster means, may

be used to rank the features. Given training feature vectors $\tilde{Z}_{i a}, i=1,2, \ldots, N_{a}$ and $\tilde{Z}_{j b}, j=1,2, \ldots, N_{b}$ from classes $a$ and $b$, respectively, the interclass separation of the $k$ th feature in the 2 classes is given by

$$
\rho_{a \rightarrow b}[k]=\frac{\left[\bar{z}_{a}(k)-\bar{z}_{b}(k)\right]^{2}}{\sum_{i=1}^{N_{a}}\left[\tilde{z}_{i a}(k)-\bar{z}_{a}(k)\right]^{2}+\sum_{j=1}^{N_{b}}\left[\tilde{z}_{j b}(k)-\bar{z}_{b}(k)\right]^{2}}, k=1,2, \ldots, D
$$

where $\bar{z}_{a}(k)$ and $\bar{z}_{b}(k)$ are the means of the $k$ th features in classes $a$ and $b$, respectively. The ranking of the features across all $C$ classes can be determined from the pair-wise ranking of $\rho_{a \rightarrow b}[k]$ for $a=1,2, \ldots, C-1 ; b=a+1, a+2, \ldots, C$. Let $r_{a \rightarrow b}[k]$ be the ranking of $\rho_{a \rightarrow b}[k]$ such that the feature with the largest inter-class separation is assigned rank 1 and the lowest is assigned $D$. Then, the inter-class rank-sum can be determined by summing the pair-wise rankings according to

$$
s(k)=\sum_{a=1}^{C-1} \sum_{b=a+1}^{C} r_{a \rightarrow b}[k], \quad k=1,2, \ldots, D .
$$

The final ranking $r(k)$ is given by Equation (7).

\section{Classification Accuracy (C-A) Criterion}

The features may also be ranked according to the classification accuracies of univariate classifiers designed for each feature. A univariate classifier is developed for each feature of the $D$-dimensional input feature vector and the classification accuracy of each feature classifier is estimated. Clearly, the training set has to be divided further into 2 sets: one to estimate the univariate classifier parameters and the other to estimate the classification accuracy. If $\alpha(k)$ is the estimated classification accuracy of the classifier for feature $\tilde{z}(k), k=1,2, \ldots, D$, the ranking of the features is given directly by ranking $\alpha(k)$. For this case

$$
r(k)=\operatorname{Rank}[\alpha(k)]
$$


is a ranking such that the feature with the highest classification accuracy is assigned rank 1 and the lowest is assigned $D$.

The ranking strategy for ordering the features using the magnitude criterion is illustrated in Figure 1 for a simulated 2-class problem with three and four 5-dimensional feature vectors in the first and second classes, respectively. That is, $C=2, D=5, N_{1}=3$, and $N_{2}=4$. The elements of vectors $R_{j c}, S_{c}, Q_{c}, S$, and $R$ in the figure are $r_{j c}(k), s_{c}(k), q_{c}(k), s(k)$, and $r(k)$, respectively.

Class $1(c=1)$

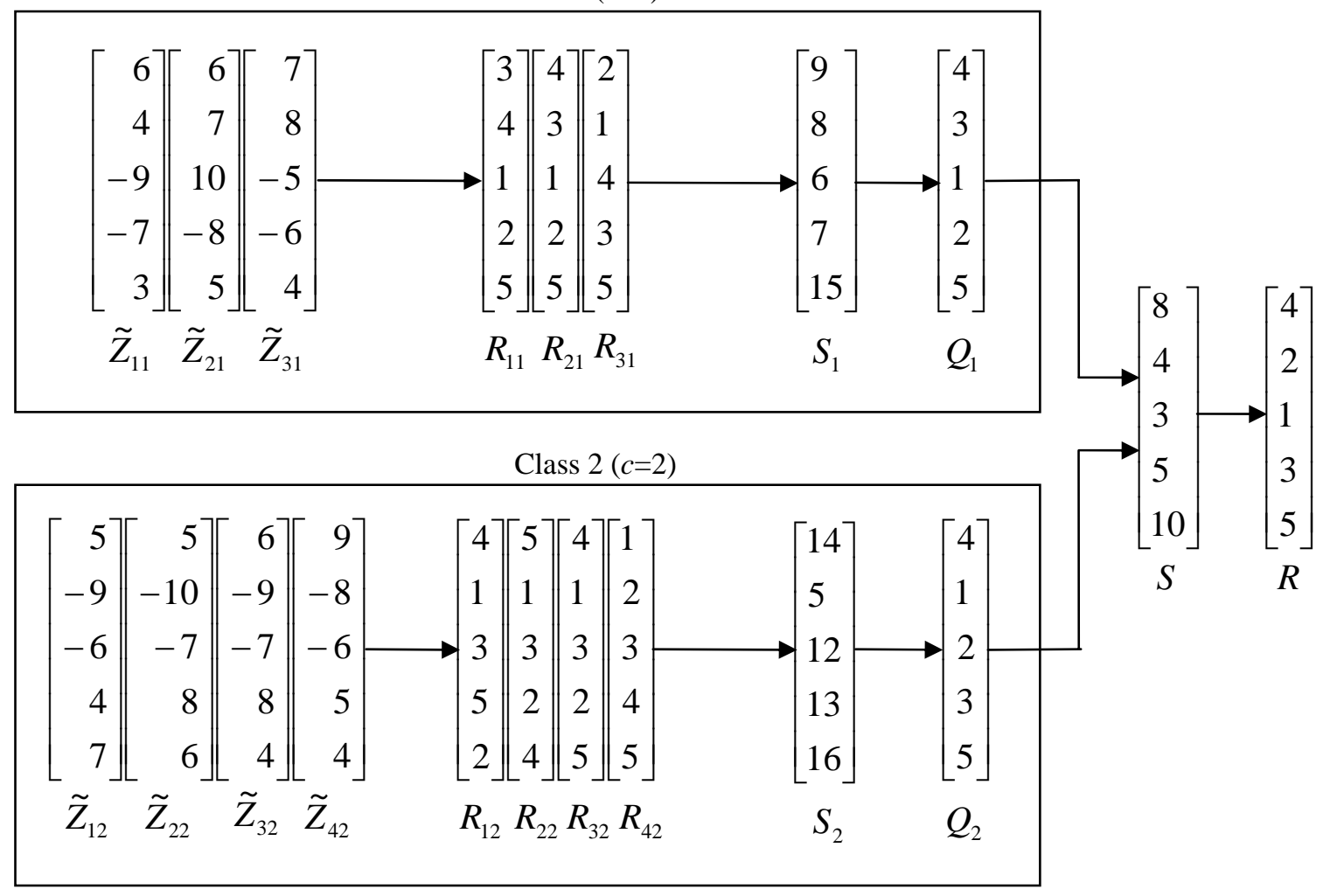

Fig. 1 Illustration of the feature ranking strategy using the magnitude criterion

\section{PCT AND DCT TRANSFORMATION MATRICES}

The features generated in Equation (1) depend on the choice of the transformation matrix $\Phi_{D}$. The specific forms of the transformation matrices for the one- and two-dimensional DCTs and issues related to determining the PCT transformation matrix are presented briefly in this section. 
$\underline{\text { DCT }}$

For the one-dimensional DCT, the elements of the transformation matrix $\Phi_{D}$ are given by

$$
\phi(u, j)=\alpha(u) \cos \left[\frac{(2 j+1) u \pi}{2 D}\right], u=0,1, \ldots,(D-1) ; j=0,1, \ldots,(D-1)
$$

where

$$
\alpha(u)=\left\{\begin{array}{c}
\sqrt{1 / D}, u=0 \\
\sqrt{2 / D}, u=1,2, \ldots,(D-1)
\end{array}\right.
$$

\section{2-Dimensional DCT}

Signals of multiple channels, such as EEGs and ERPs, can be concatenated into a multi-channel ERP vector that will have a dimension equal to $(M)(D)$, where $M$ is the number of channels and $D$ is the number of samples in each signal. Then, the same method used to decrease the dimensions for the PCT and one-dimensional DCT can be applied to decrease the dimension to facilitate multivariate classifier implementation. However, each DCT component, being a linear combination of the concatenated input, will be affected by the "artificial edges" created at the points of concatenation. The edge effects may be more severe when diverse signals from heterogeneous sensors are concatenated. Possible solutions to accommodate diverse signals include normalizing the signals from different sensors prior to DCT computation or first computing the DCTs of the signals of each channel separately and then concatenating the normalized DCTs of the $M$ channels.

Alternatively, the signal recordings of multiple channels can be observed simultaneously over time by mapping the signal activity into a 2-dimensional array $Z(m, k), m=1,2, \ldots, M ; k=1,2, \ldots, D$. To accommodate diverse multi-channel signals, the signals can be normalized. The "multichannel-time patterns" of different signal classes generate different "frequency patterns" in the transformed domain. Through coefficient selection, the 2-dimensional DCT can be used to decrease the dimension of the resulting $M \times D$ array signal space. The DCT $\tilde{Z}(u, v)$ of $Z(m, k)$ can be written as

$$
\tilde{Z}(u, v)=\Phi_{u v} Z_{M D}
$$

where $Z_{M D}$ is the $(M)(D)$-dimensional vector formed by concatenating the rows of $Z(m, k)$ and $\Phi_{u v}$ is the $(1 \times M D)$ vector formed by concatenating the rows of the 2-dimensional $(M \times D)$ basis matrix $\Phi_{u v}(m, k)$ whose elements are given by

$$
\begin{aligned}
\phi_{u v}(m, k)=\alpha(u) \alpha(v) \cos \left[\frac{(2 m+1) u \pi}{2 M}\right] \cos \left[\frac{(2 k+1) v \pi}{2 D}\right], \\
\\
\quad m=0,1, \ldots,(M-1) ; k=0,1, \ldots,(D-1)
\end{aligned}
$$


where

$$
\alpha(u)=\left\{\begin{array}{c}
\sqrt{1 / M}, u=0 \\
\sqrt{2 / M}, u=1,2, \ldots,(M-1)
\end{array} \text { and } \alpha(v)=\left\{\begin{array}{c}
\sqrt{1 / D}, v=0 \\
\sqrt{2 / D}, v=1,2, \ldots,(D-1)
\end{array}\right.\right.
$$

The DCT array $\tilde{Z}(u, v)$ may be regarded as the transformed feature array and the feature vector for multivariate classifier design can be generated by concatenating the rows of $\tilde{Z}(u, v)$ into a $(M)(D)$ dimensional vector $\tilde{Z}_{M D}$.

\section{$\underline{\text { PCT }}$}

Unlike the DCTs which are data-independent transforms, the PCT is a data-dependent transform. That is, the PCT transformation matrix has to be determined from the training sets. In Equation (1), $\Phi_{D}$ will be the PCT transformation matrix if the rows are the $D$ ordered eigenvectors (basis vectors) of the covariance matrix of $Z$. In practice, the covariance matrix is estimated from the vectors in the training set. A reliable estimate of the covariance matrix typically requires the number of training vectors to be much larger than the dimension of the vectors. Furthermore, in order to determine the $D$ eigenvectors, the number of training vectors $N$ used to estimate the covariance vector must at least exceed the dimension $D$ of the training vectors. It is, however, possible to determine a subset of the PCT basis vectors when $N<D$ using the "eigenface" approach which is widely used in face recognition applications [12]. In the eigenface approach, an estimate of the covariance matrix of $Z$ can be obtained as $\left(A A^{T}\right)$, where, $A$ is the $(D \times N)$ data matrix $\left[\left(Z_{1}-\bar{Z}\right),\left(Z_{2}-\bar{Z}\right), \ldots,\left(Z_{N}-\bar{Z}\right)\right]$ and $\bar{Z}$ is the mean of $Z_{i}, i=1,2, \ldots, N$. If $\lambda_{i}$ and $e_{i}$ are the $i^{\text {th }}$ eigenvalues and eigenvectors of $\left(A A^{T}\right)$, respectively, then it can be shown that

$$
\left(A A^{T}\right)\left(A \hat{e}_{i}\right)=\hat{\lambda}_{i}\left(A \hat{e}_{i}\right)
$$

where $\hat{\lambda}_{i}$ and $\hat{e}_{i}$ are the eigenvalues and eigenvectors of the $(N \times N)$ matrix $\left(A^{T} A\right)$, respectively. That is, $A \hat{e}_{i}, i=1,2, \ldots, N$, are the eigenvectors of $\left(A A^{T}\right)$. The advantage of this approach in practice is that the $N$ non-trivial eigenvectors of a very large $(D \times D)$ covariance matrix $\left(A A^{T}\right)$ can be determined from the eigenvectors of a much smaller $(N \times N)$ matrix $\left(A^{T} A\right)$. If $d=N$, the matrix $\hat{\Phi}_{d}$ in Equation (2) for the PCT consists of the $d$ non-trivial eigenvectors.

Note that the 1-dimensional DCT feature vector and the 2-dimensional DCT feature array have the same dimensions as their respective input signal spaces. The dimension of the PCT feature vector generated by the eigenface method is equal to the number of vectors in the training set. 


\section{GAUSSIAN SIGNAL CLASSIFICATION STRATEGY}

The formulation of the feature ranking and selection strategy developed in Section II is general, therefore, the features generated and selected can be used in conjunction with different multivariate classifiers. We, however, focus specifically on Gaussian signal classifiers because the assumption made most often is that class-conditional density functions of the feature vectors are Gaussian. This assumption is due to the analytical tractability of the Gaussian density function and also because it is an appropriate model for the case where the features are randomly corrupted versions of a single prototype feature vector [13],[14]. This section describes various forms of the multivariate Gaussian discriminant functions, issues related to determining the discriminant functions, and the resulting PCT/DCT-Gaussian multivariate classification strategy.

\section{Gaussian Discriminant Functions}

For a $C$-class classification problem, the discriminant function of the minimum-error-rate Bayes classifier, assuming Gaussian densities for the class-conditional densities, can be written as [14]

$$
g_{c}(Z)=-(1 / 2)\left(Z-\mu_{c}\right)^{T} \Psi_{c}^{-1}\left(Z-\mu_{c}\right)-(1 / 2) \ln \left|\Psi_{c}\right|+\ln P_{c} ; \quad c=1,2, \ldots, C
$$

where $Z$ is a $D$-dimensional column feature vector, $\mu_{c}$ is the $D$-dimensional mean column vector of class $c, \Psi_{c}$ is the $(D \times D)$ covariance matrix of class $c$, and $P_{c}$ is the a priori probability of class $c$. In order to implement the above discriminant function, we need the mean vectors and covariance matrices of all $C$ classes. A test pattern, represented by $Z$, is assigned to the class that yields the highest discriminant value.

If it is assumed that the covariance matrices are equal for all classes, that is, $\Psi_{c}=\Psi, c=1,2, \ldots, C$, then, Equation (20) can be written as

$$
g_{c}(Z)=-(1 / 2)\left(Z-\mu_{c}\right)^{T} \Psi^{-1}\left(Z-\mu_{c}\right)+\ln P_{c} ; \quad c=1,2, \ldots, C
$$

Furthermore, if it assumed that the prior probabilities $P_{c}$ are the same for all classes, the discriminant function in Equation (21) simplifies to

$$
g_{c}(Z)=-\left(Z-\mu_{c}\right)^{T} \Psi^{-1}\left(Z-\mu_{c}\right) ; \quad c=1,2, \ldots, C .
$$

For these two cases, we need the mean vectors of all $C$ classes and the common covariance matrix. The right hand side of Equation (22), without the minus sign, is the square of the Mahalanobis distance between $Z$ and $\mu_{c}$. Therefore, under the assumptions of equal covariance matrices and equal prior probabilities, the multivariate Gaussian classifier reduces to the nearest Mahalanobis distance classifier.

A further simplification is possible if it is assumed that the features are statistically uncorrelated and each feature has the same variance $\sigma^{2}$. That is, the covariance matrix for all of the $C$ classes is 
$\Psi_{c}=\sigma^{2} I, c=1,2, \ldots, C$, where $I$ is the identity matrix. Under these conditions the multivariate Gaussian discriminant function of Equation (22) can be written as

$$
g_{c}(Z)=-\left\|Z-\mu_{c}\right\|^{2} ; \quad c=1,2, \ldots, C
$$

which is the nearest mean Euclidean distance classifier. Only the mean vectors are required to implement this classifier.

In practice, the mean vectors and covariance matrices in the discriminant functions are estimated using the feature vectors in the training set. For the inverse of the covariance matrix to exist, the estimate of the covariance matrix must be non-singular. Therefore, it would not be possible to determine the discriminant functions in Equations (20), (21), and (22) if the covariance estimates are singular. The covariance estimate is guaranteed to be singular if the number of vectors used to estimate the covariance matrix is less than the dimension of the vectors. Therefore, if $N_{c}<D, c=1,2, \ldots C$, the covariance estimates in Equation (20) will be singular. The common covariance matrix in Equations (21) and (22) will be singular if the pooled number of training vectors does not exceed $D$, that is, if $\left(N_{1}+N_{2}+\ldots+N_{C}\right)<D$.

\section{Signal Classification Strategy}

A Gaussian classifier can be implemented by selecting the $d=N$ highest ranked features where $N$ is the number of training vectors equal to $N_{c}$ for discriminant function $g_{c}(Z)$ in Equation (20) or $\left(N_{1}+N_{2}+\ldots+N_{C}\right)$ for the discriminant functions in Equations (21) and (22). The next issue we focus on is whether all $d$ features should be used or whether there is a smaller set $L$, within the selected feature set, that may yield higher classification accuracies. By itself, this is a complex problem because $L$ is unknown and there are many different ways of choosing $L$ features out of $d$ features. To solve this problem, we systematically select $L$ highest ranked features by developing and testing classifiers for $L$ taking values $1,2,3, \ldots,(N-1)$ and then selecting the $L$ features which yield the highest classification accuracy. When $L=1$, the resulting classifier is a univariate classifier. Note that the rows of the final dimensionality reduction matrix, represented by $\hat{\Phi}_{L}$, are the $L$ basis vectors that generate the $L$ selected features.

The next two sections demonstrate the application of Gaussian multivariate classification strategy employing PCT/DCT feature ranking and selection on two signal classification problems in which the dimensionality problem is known to frequently occur. That is, the number of training vectors in the training set is less than the dimension of the input feature vectors in both classification problems. Univariate Gaussian classifiers were implemented to rank the features for the classification accuracy 
criterion. A classifier was developed independently for each subject. Half of the signals collected in the experiments were randomly selected for the training set and the test set consisted of the other half of the signals. The testing of all the signals in the test set is referred to as a trial. The classification accuracy for each trial was estimated as the ratio of the number of correctly classified signals from the test set to the total number of signals in the test set. The generation of training and test sets was repeated 200 times and the final classification accuracy was estimated by averaging the classification accuracies from the 200 trials.

\section{SINGLE CHANNEL CLASSIFICATION EXAMPLE}

In a previous study, we showed that tongue movements within the human oral cavity create unique, subtle pressure signals in the ear that can be detected using a microphone inserted into the ear-canal. It was demonstrated that the ear-pressure signals are distinct for each tongue movement and the signals can be classified accurately. Consequently, tongue movements can be mapped, via ear pressure signals, to generate control signals for HMIs without inserting any device in the oral cavity [4]. We have also demonstrated that spoken words can be recognized from the ear pressure signals [3]. Therefore, speech control signals can also be mapped via ear pressure signals into HMI control signals.

We borrowed the same data used in the previous study to demonstrate the application and evaluation of the PCT/DCT-Gaussian classification strategies developed in this paper and to compare the performances of these strategies with the results reported in the previous study. The data consisted of tongue-movement ear-pressure (TMEP) signals of 2 female and 6 male subjects corresponding to 4 tongue movement classes (Up, Down, Left, and Right). The signals were sampled at $2 \mathrm{kHz}$. Each movement was repeated at least 100 times so that 100 tongue movements could be randomly selected to represent each tongue movement class. The signals were filtered and segmented using the techniques developed in [4]. The durations of the TMEP segments were approximately $200 \mathrm{msec}$, that is 400 samples. Figure 2 shows estimates of the four tongue movements of Subject S7.
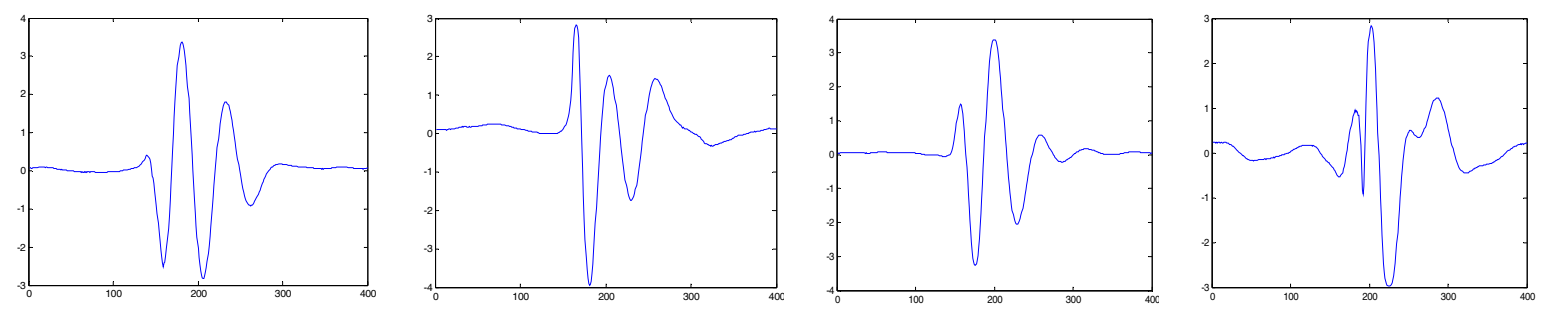

Fig. 2 The Up, Down, Left, and Right TMEP signal estimates of Subject S7 
We now assume that the covariance matrix is the same for all classes and can thus be estimated using the pooled training vectors of all 4 classes. It is also assumed that the TMEP signals are equally likely for the 4 classes so that the discriminant functions are given by Equation (22). For each subject, the training set of each class contained 50 randomly selected ear-pressure signals. Therefore, the training set to estimate the covariance matrix of the discriminant function consisted of $N=200$ signals. Consequently, the maximum dimension $d$ of the feature vector for the multivariate Gaussian classifier could not exceed 200.

\section{1-Dimensional DCT Results}

For each subject, we selected the $d=200$ highest ranked DCT coefficients out of the 400 DCT coefficients using the four different rank-based criteria developed in Section II. The performance was evaluated systematically to determine the $L$ coefficients, out of the 200 selected coefficients, that gave the best classification accuracy. A classifier was developed and evaluated for each of the following cases: the highest ranked coefficient, the 2 highest ranked coefficients, ..., the 200 highest ranked DCT coefficients. For each case, the dimension of the resulting Gaussian multivariate classifier was equal to the number of features selected. The classification accuracies for the eight subjects are shown in Table I. The best result for each subject is shown in boldface. Also included is the value of $L$, in parentheses, that gave the best result reported in the table. Each result in the table was averaged across the classification accuracies of 200 trials and each trial consisted of 200 test signals. The table also shows the results of the best classifier (a decision fusion classifier) reported in the previous study for exactly the same data [4]. The number in parenthesis for each previous result is the number of decisions fused, out of 400 , that gave the best result.

\section{PCT Results}

Using the eigenface method described in Section III, the 200 non-trivial eigenvectors were determined from the 200 vector mixture training set formed by pooling the 50-vector training sets of each of the four tongue movement classes. The transformed feature vectors $\hat{Z}$ of dimension $(200 \times 1)$ were obtained by multiplying the $(400 \times 1)$ input training vectors $Z$ by the $(200 \times 400)$ transformation matrix $\hat{\Phi}_{d}$ as in Equation (2). The features of $\hat{Z}$ were ranked to order the corresponding eigenvectors of $\hat{\Phi}_{d}$ using the 4 selection criteria. Again, the performance was evaluated systematically to determine the set of highest ranked eigenvectors that gave the best results out of the 200 ranked eigenvectors. The number in this set is also denoted by $L$. For this case, the transformation matrix $\hat{\Phi}_{L}$, therefore, had dimension $(L \times 400)$ and the transformed feature vector had dimension $(L \times 1)$. The results are also shown in Table I. 
Table I TMEP classification accuracies

\begin{tabular}{|c|c|c|c|c|c|c|c|c|c|}
\hline \multirow[t]{2}{*}{ Subject } & \multicolumn{4}{|c|}{ PCT } & \multicolumn{4}{|c|}{ 1-D DCT } & \multirow{2}{*}{$\begin{array}{c}\text { Previous } \\
\text { Results }\end{array}$} \\
\hline & Mag & Var & Int & Acc & Mag & Var & Inter & Acc & \\
\hline S1 & $\begin{array}{l}99.87 \\
(28)\end{array}$ & $\begin{array}{c}99.85 \\
(25)\end{array}$ & $\begin{array}{c}99.65 \\
(14)\end{array}$ & $\begin{array}{c}99.32 \\
\text { (5) }\end{array}$ & $\begin{array}{c}99.82 \\
(20)\end{array}$ & $\begin{array}{c}99.76 \\
(28)\end{array}$ & $\begin{array}{c}99.13 \\
(30)\end{array}$ & $\begin{array}{c}99.36 \\
(33)\end{array}$ & $\begin{array}{l}98.21 \\
(395)\end{array}$ \\
\hline S2 & $\begin{array}{c}99.78 \\
(2)\end{array}$ & $\begin{array}{c}99.77 \\
(3)\end{array}$ & $\begin{array}{c}99.78 \\
(2)\end{array}$ & $\begin{array}{c}99.80 \\
(3)\end{array}$ & $\begin{array}{c}99.73 \\
(23)\end{array}$ & $\begin{array}{c}99.62 \\
(35)\end{array}$ & $\begin{array}{c}99.55 \\
(36)\end{array}$ & $\begin{array}{c}99.56 \\
(52)\end{array}$ & $\begin{array}{l}96.52 \\
(400)\end{array}$ \\
\hline S3 & $\begin{array}{c}99.26 \\
(8)\end{array}$ & $\begin{array}{c}99.27 \\
(9)\end{array}$ & $\begin{array}{c}99.23 \\
(11)\end{array}$ & $\begin{array}{c}99.21 \\
(6)\end{array}$ & $\begin{array}{c}99.33 \\
(48) \\
\end{array}$ & $\begin{array}{c}99.27 \\
(46)\end{array}$ & $\begin{array}{c}99.21 \\
(39)\end{array}$ & $\begin{array}{c}99.23 \\
(43)\end{array}$ & $\begin{array}{l}98.39 \\
(304)\end{array}$ \\
\hline S4 & $\begin{array}{c}99.95 \\
(9)\end{array}$ & $\begin{array}{c}99.94 \\
(9)\end{array}$ & $\begin{array}{c}99.89 \\
(19)\end{array}$ & $\begin{array}{c}99.83 \\
(9)\end{array}$ & $\begin{array}{l}99.76 \\
(26)\end{array}$ & $\begin{array}{c}99.77 \\
(27) \\
\end{array}$ & $\begin{array}{c}99.81 \\
(35)\end{array}$ & $\begin{array}{c}99.84 \\
(16)\end{array}$ & $\begin{array}{l}99.74 \\
(400)\end{array}$ \\
\hline S5 & $\begin{array}{c}100.0 \\
(12)\end{array}$ & $\begin{array}{c}100.0 \\
(12)\end{array}$ & $\begin{array}{c}99.96 \\
(9) \\
\end{array}$ & $\begin{array}{c}99.90 \\
(7)\end{array}$ & $\begin{array}{c}100.0 \\
(15)\end{array}$ & $\begin{array}{c}100.0 \\
(19)\end{array}$ & $\begin{array}{c}99.96 \\
(21) \\
\end{array}$ & $\begin{array}{c}99.99 \\
(27) \\
\end{array}$ & $\begin{array}{l}94.06 \\
(400) \\
\end{array}$ \\
\hline S6 & $\begin{array}{c}99.95 \\
(8) \\
\end{array}$ & $\begin{array}{c}99.96 \\
(8)\end{array}$ & $\begin{array}{c}99.91 \\
(5)\end{array}$ & $\begin{array}{c}99.83 \\
(8) \\
\end{array}$ & $\begin{array}{c}99.96 \\
(35) \\
\end{array}$ & $\begin{array}{c}99.96 \\
(35)\end{array}$ & $\begin{array}{c}99.89 \\
(38)\end{array}$ & $\begin{array}{c}99.91 \\
(21)\end{array}$ & $\begin{array}{l}93.52 \\
(400)\end{array}$ \\
\hline S7 & $\begin{array}{c}100.0 \\
(3)\end{array}$ & $\begin{array}{c}100.0 \\
(5)\end{array}$ & $\begin{array}{c}100.0 \\
(11)\end{array}$ & $\begin{array}{c}100.0 \\
(4)\end{array}$ & $\begin{array}{c}100.0 \\
(16)\end{array}$ & $\begin{array}{c}100.0 \\
(19)\end{array}$ & $\begin{array}{c}100.0 \\
(17)\end{array}$ & $\begin{array}{c}100.0 \\
(28)\end{array}$ & $\begin{array}{l}99.60 \\
(345)\end{array}$ \\
\hline S8 & $\begin{array}{c}99.97 \\
\text { (5) }\end{array}$ & $\begin{array}{c}99.97 \\
(5)\end{array}$ & $\begin{array}{c}99.78 \\
\text { (5) }\end{array}$ & $\begin{array}{c}99.92 \\
(4)\end{array}$ & $\begin{array}{c}99.91 \\
(18)\end{array}$ & $\begin{array}{c}99.85 \\
(17)\end{array}$ & $\begin{array}{c}99.28 \\
(9)\end{array}$ & $\begin{array}{c}99.72 \\
(12)\end{array}$ & $\begin{array}{l}96.30 \\
(400)\end{array}$ \\
\hline Average & 99.85 & 99.84 & 99.77 & 99.73 & 99.81 & 99.78 & 99.6 & 99.70 & 97.04 \\
\hline
\end{tabular}

\section{MULTI-CHANNEL SIGNAL CLASSIFICATION EXAMPLE}

The second set of experiments involved classifying multi-channel match-mismatch ERPs of three subjects borrowed from another previous classification study [15]. This ERP data set was selected because the curse of dimensionality is quite severe and also because comparisons can be made with the classification results obtained in the previous study. A brief description of the ERP experiments is provided in this paragraph to understand the classification problem and to note the occurrence of the dimensionality problem. The goal of the previous study was to show that ERPs can reliably identify when a match occurs between what a subject thinks and sees. The ERPs were collected from individuals engaged in making explicit match/mismatch comparisons between $C=2$ sequentially presented stimuli. The data set consisted of 14-channel ERPs of 1 female (A1:16 years) and 2 male (A2:21 and A3:23 years) subjects. The subjects were instructed to "think" about the first video stimulus (picture of an object) and then to respond whether the next video stimulus (printed word of an object) matched or did not match the first stimulus in "meaning." The period between the first and second stimulus varied randomly between 2 to 5 seconds. The response from each channel, time-locked to the onset of the second stimulus, was recorded as a match category or mismatch category depending on which of 2 keys was pressed by the subject. ERP data were collected from electrodes placed on the frontal left-hemisphere (F7, F3), right-hemisphere (F8), and midline (Fz); on the temporal left-hemisphere (T3, T5) and right-hemisphere (T4, T6); on the central left-hemisphere (C3) and central right-hemisphere (C4); and on the parietal left-hemisphere (P3), righthemisphere (P4), and midline (Pz). The 14 electrodes were referenced to linked earlobe leads. The electrooculogram (EOG) was also recorded with two electrodes placed lateral and below the left eye 
(bipolar montage). Single-trial ERPs were digitized over $1 \mathrm{sec}$ using a $10 \mathrm{msec}$ sampling period beginning $100 \mathrm{msec}$ prior to stimulus onset. The 10 samples corresponding to the prestimulus period were removed, thus, the dimension of the single-trial ERPs was $K=90$. Trials in which the peak-to-peak amplitude exceeded $100 \mu V$ in any one electrode channel or $50 \mu V$ in the eye channel were regarded as contaminated by artifacts and rejected. In order to accommodate the different amplitudes across the channels, each single-trial ERP was scale normalized by dividing the samples of the ERP by the standard deviation of the ERP samples. Additionally, each single-trial ERP was de-trended to remove slope variations in the ERPs within and across the channels. From the ensembles collected, an equal number of artifact-free single-trial match and mismatch ERPs were selected for each subject, however, the number varied across the subjects. The total number of 14-channel single-trial ERP arrays collected for each category was 71, 71, 82 from subjects A1, A2, and A3, respectively. Figure 3 shows the match and mismatch array estimates obtained by averaging the single-trial ERPs of Subject A1.
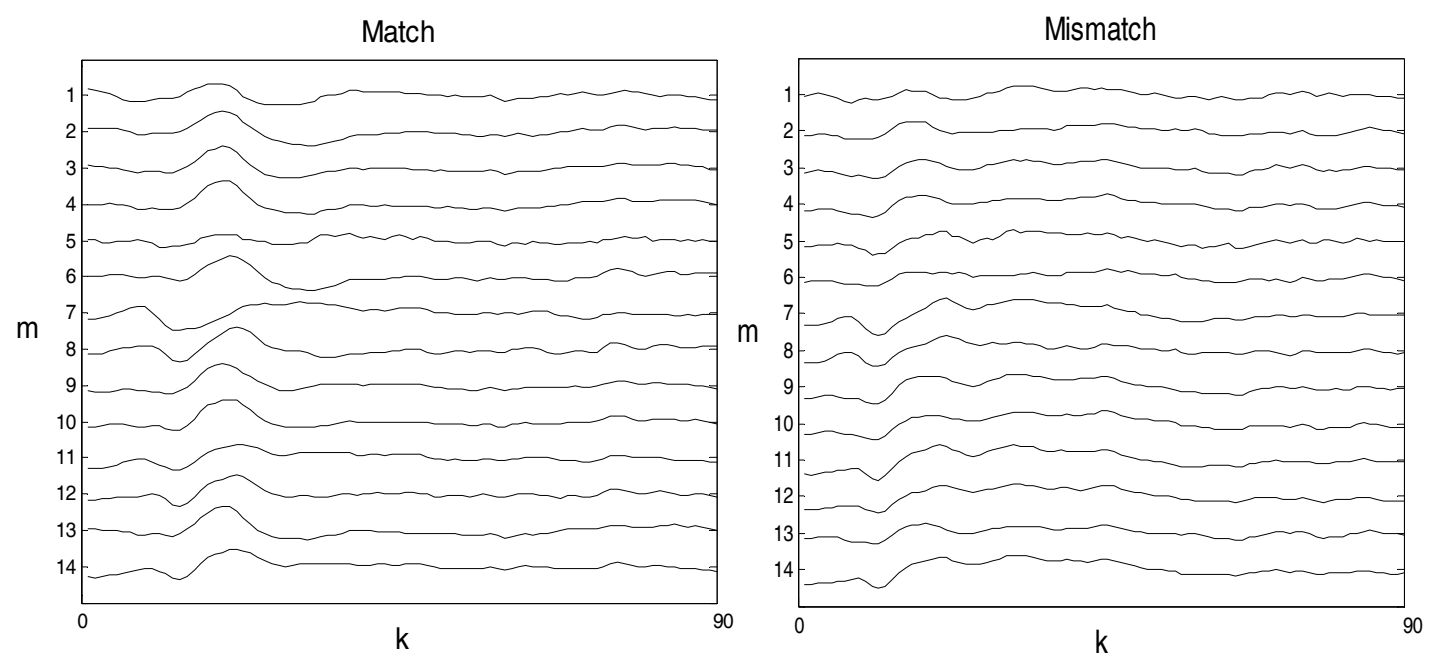

Fig. 3 The ensemble averaged match and mismatch ERP arrays of Subject A1

In the usual ERP model, it is assumed that the background EEG noise is statistically equivalent across the ERP classes [15],[16]. Consequently, it can be assumed that the covariance matrix is the same across the match and mismatch conditions. Therefore, the covariance matrix can be estimated from the pooled training sets of the 2 classes. The match and mismatch conditions were equally likely, therefore, the discriminant functions are given by Equation (22). The training set for each class contained 35 ERP arrays (approximately half). Given that each training array contributed a training vector, the dimension $d$ of the transformed feature vector could not exceed 70 for the resulting covariance matrix in Equation (22) 
to be non-singular. That is, the dimension of the input feature had to be decreased from $90 \times 14=1260$ to a maximum of 70 in order to implement the discriminant function in Equation (22).

Signal averaging is the most often used operation to improve the signal-to-noise ratios in ERPs [15],[16] and classification results can be presented as a function of the number of single-trials averaged. The classification accuracies are presented for single-trial ERPs and for ERPs averaged across $r=2$ and 4 single-trials. The results derived in [15] were used to estimate the covariance matrix of averaged ERPs from single-trial ERPs. From the linearity property, the DCT of ERPs averaged across $r$ trials is equal to average of the DCT of the $r$ ERPs. Therefore, in order to facilitate multivariate classifier development based on the DCT coefficients, the averaging operation was performed in the DCT domain instead of averaging in the discrete-time domain.

\section{2-dimensional DCT Results}

In order to develop a multivariate classifier that uses the 2-dimensional DCT coefficients for features, the maximum number of DCT coefficients selected from the $M \times D$ coefficient array had to be less than the pooled number of training ERP arrays. In the ERP experiments, $D$ was $90, M$ was 14 , and the number of pooled training ERP arrays was 70. Therefore, the 1260 DCT coefficients were ranked using the 4 different criteria and the 70 coefficients with the highest ranks were selected according to each criterion. The performance was evaluated systematically to determine the coefficients, out of the 70 , that gave the best results. As before, the dimensions of the resulting multivariate classifiers were equal to the number of coefficients selected. The classification accuracies for the ERPs of the 3 subjects, averaged across 200 trials, are summarized in Table II. The best results from the previous study for the 3 subjects, using a weighted multi-channel ERP fusion technique [16], are included in the last column of the table. Because the previous study did not include single-trial classification, the results for $r=1$ are labeled NA.

\section{1-dimensional DCT and PCT Results}

As noted in Section III, the signals of the $M$ channels can be concatenated into a multi-channel ERP data fusion vector that will have a dimension equal to $(D)(M) \times 1$. Then, the PCT and one-dimensional DCT dimensionality reduction techniques can be applied to decrease the dimension of the resulting multichannel ERP vector to satisfy the dimensionality conditions. The dimension of the concatenated vector was decreased from 1260 to 70 by selecting the basis vectors according to the four criteria. Gaussian multivariate classifiers were developed and evaluated to determine the set of coefficients that gave the best result for each selection criterion. The one-dimensional DCT and PCT classification accuracies for the ERPs are also included in Table II. Again, the value of $L$ that gave the best result is shown in parentheses. 
Table II ERP classification accuracies

\begin{tabular}{|c|c|c|c|c|c|c|c|c|c|c|c|c|c|c|}
\hline \multirow{2}{*}{$\begin{array}{c}\text { Sub- } \\
\text { ject }\end{array}$} & \multirow[b]{2}{*}{$\mathbf{r}$} & \multicolumn{4}{|c|}{ 2-D DCT } & \multicolumn{4}{|c|}{ 1-D DCT } & \multicolumn{4}{|c|}{ PCT } & \multirow{2}{*}{$\begin{array}{l}\text { Previous } \\
\text { Results }\end{array}$} \\
\hline & & MAG & VAR & I-S & C-A & MAG & VAR & I-S & C-A & MAG & VAR & I-S & C-A & \\
\hline \multirow{3}{*}{ A1 } & 1 & $\begin{array}{c}72.89 \\
(12)\end{array}$ & $\begin{array}{c}72.89 \\
(8)\end{array}$ & $\begin{array}{c}73.25 \\
(26)\end{array}$ & $\begin{array}{c}71.43 \\
(30)\end{array}$ & $\begin{array}{c}73.21 \\
(37)\end{array}$ & $\begin{array}{c}72.75 \\
(23)\end{array}$ & $\begin{array}{c}74.21 \\
(4)\end{array}$ & $\begin{array}{c}73.18 \\
(25)\end{array}$ & $\begin{array}{c}77.33 \\
(33)\end{array}$ & $\begin{array}{c}78.87 \\
\text { (7) }\end{array}$ & $\begin{array}{c}76.00 \\
(15)\end{array}$ & $\begin{array}{c}73.21 \\
(37)\end{array}$ & NA \\
\hline & 2 & $\begin{array}{c}78.84 \\
(66)\end{array}$ & $\begin{array}{c}77.63 \\
(42) \\
\end{array}$ & $\begin{array}{c}79.38 \\
(31) \\
\end{array}$ & $\begin{array}{c}76.45 \\
(26)\end{array}$ & $\begin{array}{c}81.83 \\
(37) \\
\end{array}$ & $\begin{array}{c}80.04 \\
(61) \\
\end{array}$ & $\begin{array}{c}80.77 \\
(34) \\
\end{array}$ & $\begin{array}{c}79.30 \\
(22) \\
\end{array}$ & $\begin{array}{c}85.82 \\
(35) \\
\end{array}$ & $\begin{array}{c}86.57 \\
(19) \\
\end{array}$ & $\begin{array}{c}83.72 \\
(5)\end{array}$ & $\begin{array}{c}83.56 \\
(7) \\
\end{array}$ & 82.51 \\
\hline & 4 & $\begin{array}{c}88.31 \\
(65) \\
\end{array}$ & $\begin{array}{c}88.14 \\
(43)\end{array}$ & $\begin{array}{c}89.49 \\
(20)\end{array}$ & $\begin{array}{c}86.22 \\
(28)\end{array}$ & $\begin{array}{c}89.23 \\
(36) \\
\end{array}$ & $\begin{array}{c}87.54 \\
(59) \\
\end{array}$ & $\begin{array}{c}88.74 \\
(9) \\
\end{array}$ & $\begin{array}{c}88.05 \\
(21) \\
\end{array}$ & $\begin{array}{c}94.36 \\
(13) \\
\end{array}$ & $\begin{array}{c}94.23 \\
(21) \\
\end{array}$ & $\begin{array}{c}92.96 \\
(5)\end{array}$ & $\begin{array}{c}92.91 \\
(7)\end{array}$ & 91.33 \\
\hline \multirow{3}{*}{ A2 } & 1 & $\begin{array}{c}73.13 \\
(14)\end{array}$ & $\begin{array}{c}71.37 \\
(59)\end{array}$ & $\begin{array}{l}69.37 \\
(33)\end{array}$ & $\begin{array}{c}65.07 \\
(17)\end{array}$ & $\begin{array}{c}72.80 \\
(50)\end{array}$ & $\begin{array}{c}72.87 \\
(39)\end{array}$ & $\begin{array}{c}70.30 \\
(36)\end{array}$ & $\begin{array}{c}68.80 \\
(20)\end{array}$ & $\begin{array}{c}74.94 \\
(51)\end{array}$ & $\begin{array}{c}76.75 \\
(67) \\
\end{array}$ & $\begin{array}{c}73.06 \\
(41) \\
\end{array}$ & $\begin{array}{c}72.63 \\
(51) \\
\end{array}$ & $\mathrm{N}$ \\
\hline & 2 & $\begin{array}{c}78.97 \\
(23) \\
\end{array}$ & $\begin{array}{c}77.33 \\
(52) \\
\end{array}$ & $\begin{array}{l}74.91 \\
(25)\end{array}$ & $\begin{array}{c}71.21 \\
(33)\end{array}$ & $\begin{array}{c}78.05 \\
(20)\end{array}$ & $\begin{array}{c}76.07 \\
(20) \\
\end{array}$ & $\begin{array}{c}74.68 \\
(28) \\
\end{array}$ & $\begin{array}{c}75.02 \\
(28) \\
\end{array}$ & $\begin{array}{c}85.09 \\
(53)\end{array}$ & $\begin{array}{c}85.22 \\
(44)\end{array}$ & $\begin{array}{c}82.38 \\
(44)\end{array}$ & $\begin{array}{l}82.35 \\
(29) \\
\end{array}$ & 72.09 \\
\hline & 4 & $\begin{array}{c}88.32 \\
(15)\end{array}$ & $\begin{array}{c}86.63 \\
(52)\end{array}$ & $\begin{array}{c}84.95 \\
(27)\end{array}$ & $\begin{array}{c}79.06 \\
(33)\end{array}$ & $\begin{array}{c}88.57 \\
(18)\end{array}$ & $\begin{array}{c}85.64 \\
(14)\end{array}$ & $\begin{array}{c}84.19 \\
(26)\end{array}$ & $\begin{array}{c}82.81 \\
(41)\end{array}$ & $\begin{array}{c}93.81 \\
(43)\end{array}$ & $\begin{array}{c}91.68 \\
(47)\end{array}$ & $\begin{array}{l}91.50 \\
(19)\end{array}$ & $\begin{array}{c}88.57 \\
(18)\end{array}$ & 31.19 \\
\hline \multirow{3}{*}{ A3 } & 1 & $\begin{array}{c}71.47 \\
(47)\end{array}$ & $\begin{array}{c}71.24 \\
(50)\end{array}$ & $\begin{array}{c}68.00 \\
(2)\end{array}$ & $\begin{array}{c}65.59 \\
(38)\end{array}$ & $\begin{array}{c}72.24 \\
(60)\end{array}$ & $\begin{array}{c}74.38 \\
(54)\end{array}$ & $\begin{array}{c}69.68 \\
(6)\end{array}$ & $\begin{array}{c}68.06 \\
(23)\end{array}$ & $\begin{array}{c}75.71 \\
(65)\end{array}$ & $\begin{array}{c}75.91 \\
(63)\end{array}$ & $\begin{array}{c}72.82 \\
(58)\end{array}$ & $\begin{array}{c}72.29 \\
(62)\end{array}$ & NA \\
\hline & 2 & $\begin{array}{c}79.45 \\
(56) \\
\end{array}$ & $\begin{array}{r}80.67 \\
(58)\end{array}$ & $\begin{array}{c}77.65 \\
(2)\end{array}$ & $\begin{array}{l}71.64 \\
(34)\end{array}$ & $\begin{array}{l}81.00 \\
(62)\end{array}$ & $\begin{array}{c}84.49 \\
(54) \\
\end{array}$ & $\begin{array}{c}77.90 \\
(8) \\
\end{array}$ & $\begin{array}{c}74.02 \\
(17) \\
\end{array}$ & $\begin{array}{c}85.58 \\
(65)\end{array}$ & $\begin{array}{c}83.93 \\
(46)\end{array}$ & $\begin{array}{c}82.80 \\
(48)\end{array}$ & $\begin{array}{c}82.44 \\
(45)\end{array}$ & 76.63 \\
\hline & 4 & $\begin{array}{c}84.78 \\
(53)\end{array}$ & $\begin{array}{c}85.13 \\
(58)\end{array}$ & $\begin{array}{l}79.30 \\
\text { (3) }\end{array}$ & $\begin{array}{c}77.01 \\
(20)\end{array}$ & $\begin{array}{l}88.60 \\
(63)\end{array}$ & $\begin{array}{c}91.19 \\
(56)\end{array}$ & $\begin{array}{c}86.84 \\
\text { (7) }\end{array}$ & $\begin{array}{c}81.96 \\
(13)\end{array}$ & $\begin{array}{c}91.35 \\
(57)\end{array}$ & $\begin{array}{c}91.75 \\
(43)\end{array}$ & $\begin{array}{c}88.93 \\
(25)\end{array}$ & $\begin{array}{c}88.58 \\
(41)\end{array}$ & 4.78 \\
\hline
\end{tabular}

\section{ANALYSES OF RESULTS}

This section presents an analysis of the results from the two sets of classification experiments in order to determine which combinations of the PCT, DCT, and feature selection criteria give the best classification accuracies and to also compare the results with those reported in the previous studies. Furthermore, the significant reduction in dimension using the PCT and DCT is also noted.

\section{TMEP Signal Classification}

The PCT and DCT approaches in conjunction with the four basis vector selection criteria resulted in 8 classifiers for the TMEP signal classification problems. The PCT classifier using the magnitude selection criterion gave the best results averaged across the 8 subjects. Note, however, that the average classification accuracies of all 8 classifiers were greater than or equal to $99.6 \%$ and the difference between the best and worst classifier was only $0.25 \%$. Because all 8 classifiers gave very good results, it is difficult to convincingly pick the best classifier from this set of experiments. On the average, the PCT classifiers gave better results than the DCT classifiers across all 4 coefficient selection criteria.

In the previous work reported in [4], exactly the same TMEP signals were classified using a decision fusion classifier, a matched filter, a Gaussian classifier using AR model parameters, and a nonlinear alignment classifier. It was shown that, in general, the best results were obtained using the decision fusion classifier. The decision fusion classifier combined the decisions of univariate classifiers designed 
for each TMEP signal sample. The univariate decisions of the test TMEP signal samples were fused into a decision fusion vector and the resulting decision fusion vector was classified using a discrete Bayes classifier to determine the final class of the test TMEP signal. Although there was very little room for improvement, the classification accuracies obtained using the PCT- and DCT-Gaussian classifiers developed in this paper are consistently higher than those obtained using the decision fusion classifier and thus also higher than those obtained using the matched filter, the Gaussian classifier using AR model parameters, and the non-linear alignment classifier.

\section{ERP Signal Classification}

For the ERP classification experiments, the 2-dimensional DCT, 1-dimensional DCT, and the PCT, in conjunction with the 4 selection criteria resulted in 12 classifiers. Table III shows the classification accuracies averaged across the 3 subjects as a function of $r$. On the average, the PCT gave the best classification accuracies and the 2-dimensional DCT gave the worst accuracies across the 4 selection criteria and as well as $r$. The classification accuracy of the PCT classifiers using the magnitude criterion was slightly higher than that of the PCT classifier using the variance selection criteria for $r=2$ and 4 .

Table III Classification accuracies averaged across the 3 subjects as a function of $r$

\begin{tabular}{|c|l|l|l|l|l|l|l|l|c|c|c|c|}
\hline \multirow{2}{*}{$\mathbf{r}$} & \multicolumn{4}{|c|}{ 2-D DCT } & \multicolumn{4}{|c|}{ 1-D DCT } & \multicolumn{4}{c|}{ PCT } \\
\cline { 2 - 13 } & MAG & VAR & I-S & C-A & MAG & VAR & I-S & C-A & MAG & VAR & I-S & C-A \\
\hline 1 & 72.5 & 71.83 & 70.21 & 67.36 & 72.75 & 73.33 & 71.4 & 70.01 & 76 & $\mathbf{7 7 . 1 1}$ & 73.96 & 73.42 \\
\hline 2 & 79.1 & 78.54 & 77.31 & 73.1 & 80.29 & 80.2 & 77.78 & 76.11 & $\mathbf{8 5 . 5}$ & 85.24 & 82.97 & 82.78 \\
\hline 4 & 87.14 & 86.63 & 84.58 & 80.76 & 88.8 & 88.12 & 86.59 & 84.27 & $\mathbf{9 3 . 1 7}$ & 92.55 & 91.13 & 91.01 \\
\hline
\end{tabular}

In the previous decision and data fusion study [16] involving the same multi-channel ERP data, the best results were obtained using a data fusion strategy in which the ERPs of all 14 channels were concatenated into a multi-channel ERP vector. In order to develop a multivariate classifier, the dimension of the multi-channel ERP vector was decreased to satisfy the dimensionality condition using a two-step procedure. In the first step, elements with small inter-class separations were discarded. The most correlated elements in the remaining set were combined systematically, in a pairwise fashion, to decrease the dimension further in the second step. The features selected were the discrete Karhunen-Loeve transform linear combinations of the ERP samples of the reduced-dimension multi-channel ERP vectors. The classification accuracies of the PCT classifiers using the variance and magnitude criteria are consistently higher, especially for Subjects A2 and A3, than the accuracies reported in the previous study.

The ERP classification accuracies were not as high as the TMEP classification accuracies. This, however, is not unexpected because ERPs are known to have very poor SNRs [15],[16]. In a relative sense, TMEP signals are not as noisy as ERP signals. We can, therefore, conclude that there is very little 
difference between the performances of the PCT and DCT classifiers for the high SNR case. The PCT classifiers gave better results than the DCT classifiers for ERPs, that is, for the small SNR case. Considering both sets of experiments together, the PCT classifiers using the maximum magnitude and maximum variance criteria yielded the highest classification accuracies.

The results in Tables I and II show that higher classification accuracies, for both the PCT and DCT, are obtained by selecting an even smaller set $L$ from the initial set of $d$ basis vectors. Similar results in which the classifier performance increases initially as features are added but diminishes as more features are added have been reported in work related to the classification of myoelectric signals [17]. The ranking strategy, therefore, serves two purposes: to overcome the curse of dimensionality in order to facilitate multivariate classifier implementation and to also facilitate the selection of a smaller set of features, from the rank-ordered feature set, which give the best performance.

Also interesting to note is that only a small number of linear combinations of the input are needed to obtain the best classification results. This is due to the high compaction of information into a small set of PCT and DCT transform coefficients. Therefore, a large number of PCT and DCT coefficients can be dropped to decrease the dimensionality significantly in order to facilitate multivariate classifier development. The variations in $L$ from subject to subject in the results presented are very likely due to the expected differences in the signals generated by different individuals.

Comparing classifiers based on classification accuracies is a common practice. The classifiers developed in this study are superior to those in our previous studies in the sense that higher classification accuracies are obtained on exactly the same data using exactly the same methods to generate the training and test sets. For each subject, an unequal variance pairwise t-test showed that the differences between the classification accuracies of the best PCT/DCT-Gaussian classifier and the classification accuracies of the best classifiers from the previous study are statistically significant $(\mathrm{p}<0.0011)$ even though the mean differences are quite small. However, the standard deviations are also quite small, especially when the average classification accuracies are very high. Moreover, the classification accuracy is degenerate (zero standard deviation) when the classification accuracy is $100 \%$. The t-test results should also be interpreted with caution because the assumption for normality was violated in all cases. The Wilcoxon rank-sum test also yielded p-values comparable to those of paired t-tests except for subject S3 ( $p=0.6568$ ). The standard deviations of both pairs of classification accuracies of subject S3 were relatively high. Paired t-tests and Wilcoxon rank-sum tests showed that the differences between the previous and the best DCT and PCT classification accuracies for the ERP signals, shown in Table II, were also statistically significant $(\mathrm{p}<0.0011)$ for all three subjects. 


\section{CONCLUSIONS}

The feature ranking and selection strategy developed in this paper offers a means to overcome the curse of dimensionality frequently encountered in multivariate signal classification due to the large input signal spaces and the practical difficulties associated with collecting large signal sets. The strategy not only facilitates dimensionality reduction for multivariate classifier implementation but also offers a means to systematically select, from the rank-ordered feature set, a smaller set of features that yield the best classification accuracies. Through the two sets of classification experiments, it was shown that the reduction in the dimensions of the transformed PCT and DCT feature spaces was quite significant. It was also shown that the classification accuracies of the resulting PCT- and DCT-Gaussian signal classifiers were significantly higher than those we had reported in our previous work involving the classification of the same signal sets.

Finally, it is concluded that the feature ranking and selection based dimensionality reduction strategy developed in this paper makes it possible to implement multivariate classifiers without having to collect a very large number of training signals simply to satisfy the dimensionality conditions. Consequently, the PCT- and DCT-Gaussian classification strategies can be beneficial for designing personalized human-machine-interface (HMI) signal classifiers for individuals from whom only a limited number of training signals can reliably be collected due to severe disabilities.

\section{ACKNOWLEDGEMENTS}

The authors would like to thank the reviewers for their comments and suggestions. The authors would also like to gratefully acknowledge that this work was partially supported by the following grants: NIH RO1 HD17860; NSF BNS 82-10846; NIH 1R43HD042367-01A1, and UK Engineering and PhysicalSciences Research Council Grant EP/F01869X/1.

\section{REFERENCES}

[1] H. Medicherla, and A. Sekmen, "Human-robot interaction via voice-controllable intelligent user interface,” Robotica, vol. 25, no. 5, pp. 521-527, Sep. 2007.

[2] H. G. Nik, G. M. Gutt, and N. Peixoto, "Voice recognition algorithm for portable assistive devices,” Sensors, pp. 997-1000, 28-31 Oct. 2007.

[3] R.Vaidyanathan, M.Fargues, R.S. Kurkan, L. Gupta, S. Kota, R.D. Quinn, \& D. Lin, “A dualmode human-robot teleoperation interface based on airflow in the aural cavity,” International Journal of Robotics Research, Special Issue, 1205-1223, November 2007. 
[4] R. Vaidyanathan, B. Chung, L. Gupta, H. Kook, S. Kota, \& J. West, “A tongue-movement communication and control strategy for hands-free human-machine interfaces," IEEE Transactions on Systems, Man, \& Cybernetics - A, vol. 37, No. 4, 533-546, July 2007.

[5] O. Fukuda, T. Tsuji, M. Kaneki, and A. Otsuka, "A human-assisting manipulator teleoperated by EMG signals and arm motions," IEEE Transactions on Robotics and Automation, vol. 19, no. 2, pp. 210-222, April. 2003.

[6] A. Ferreira, W. C. Celeste, F. A. Cheein, T. F. Bastos-Filho, M. Sarcinelli-Filho, and R. Carelli, "Human-machine interface based on EMG and EEG applied to robotic systems," Journal of NeuroEngineering and Rehabilitation, vol. 5, March. 2008.

[7] K. Tanaka, K. Matsunaga, and H. Wang, "Electroencephalogram-based control of an electric wheelchair." IEEE Transactions on Robotics, vol. 21, no. 4, pp. 762-766, Aug. 2005.

[8] J. D. R. Millán, F. Renkens, J. Mouriño, and W. Gerstner, "Noninvasive brain-actuated control of a mobile robot by human EEG." IEEE Transactions on Biomedical Engineering, vol. 51, no. 6, pp. 1026-1033, June. 2004.

[9] L.G. Cohen and N. Birbaumer, "The physiology of brain-computer interfaces," Journal of Physiology, Vol. 579(3), 570, 2007.

[10] L. Gupta and S. Ma, "Gesture-based interaction and communication: automated classification of hand gesture contours,” IEEE Transactions on Systems, Man, \& Cybernetics - C, vol. 31, No. 1, 114-120, 2001.

[11] M. Urban, and P. Bajcsy, "Fusion of voice, gesture, and human-computer interface controls for remotely operated robot." Proceedings of the $8^{\text {th }}$ International Conference on Information Fusion, vol. 2, July 25-July 28, 2005.

[12] M. Turk, A. Pentland, “Eigenfaces for recognition”, Journal of Congnitive Neuroscience, vol. 3, no. 1, pp. 71-86, 1991.

[13] G. J. McLachlan, Disciminant Analysis and statistical pattern recognition, John Wiley, New York, 1992.

[14] R. O. Duda, P. E. Hart, D. G. Stork, "Pattern Classification”, $2^{\text {nd }}$ ed., John Wiley \& Sons. Inc, New York, 2001.

[15] L. Gupta, J. Phegley, \& D.L. Molfese, "Parametric classification of multichannel evoked potentials," IEEE Transactions on Biomedical Engineering, vol. 49, no. 8, pp. 905-911, Aug. 2002 (vol. 49, no. 9, 1070, September 2002).

[16] L. Gupta, B. Chung, M.D. Srinath, D.L. Molfese, \& H. Kook "Multi-channel fusion models for the parametric classification of differential brain activity,” IEEE Transactions on Biomedical Engineering, Vol. 52, No. 11, 1869-1881, 2005. 
[17] K. Englehart, B. Hudgin, \& P.A. Parker, “A wavelet-based continuous classification scheme for multifunction myoelectric control,” IEEE Transactions on Biomedical Engineering, Vol. 48, 302311, 2001. 


\section{Figure Captions}

Fig. 1 Illustration of the feature ranking strategy using the magnitude criterion

Fig. 2 The Up, Down, Left, and Right TMEP signal estimates of Subject S7

Fig. 3 The ensemble averaged match and mismatch ERP arrays of Subject A1 


\section{Table Captions}

Table I TMEP classification accuracies

Table II ERP classification accuracies

Table III Classification accuracies averaged across the 3 subjects as a function of $r$ 


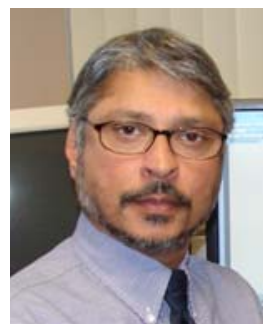

Lalit Gupta received the B.E. (Hons) degree in electrical engineering from the Birla Institute of Technology and Science, Pilani, India (1976), the M.S. degree in digital systems from Brunel University, Middlesex, England (1981), and the Ph.D degree in electrical engineering from Southern Methodist University, Dallas, Texas (1986).

He is currently a Professor of Electrical and Computer Engineering at Southern Illinois University, Carbondale, Illinois. His research interests are in pattern recognition, neuroinformatics, neural networks, and signal processing. He has been awarded contracts from the Army Research office to conduct research in the development of smart munitions, from Seagate Technology on image compression research, from Cleveland Medical Devices on NIH funded projects related to brain wave form analysis and classification, from Think-A-Move Inc. on a NIH funded project related to Human-Machine Interfacing, from Neuronetrix on a NIH funded project on detecting neurological disorders from evoked potentials, and from the Naval Postgraduate School on research related to Human-Machine Interfacing.

Dr. Gupta has numerous publications in the areas of neural networks, evoked potential analyses and classification, and multi-channel/sensor information fusion strategies. Dr. Gupta serves as an Associate Editor of the Pattern Recognition Journal and is a Senior Member of the IEEE.

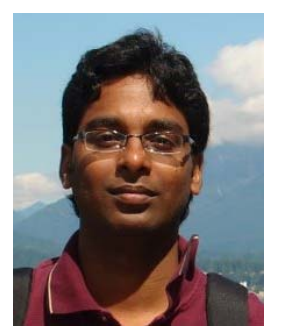

Srinivas Kota received the B.E. degree in electronics and communications engineering from Andhra University, Visakhapatnam, India, in 2000 and the M.S. degree in electrical engineering from Southern Illinois University, Carbondale, in 2004. He is currently working toward the Ph.D. degree in the Department of Electrical and Computer Engineering, Southern Illinois University.

His research interests are in signal processing, image processing, pattern recognition and neuroinformatics.

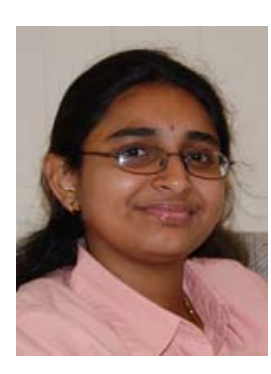

Swetha Murali received the B.E. degree in Instrumentation and Control Engineering from Anna University, Chennai, India (2006) and the M.S. degree in Electrical and Computer Engineering from Southern Illinois University, Carbondale, Illinois (2008). She is currently working for the Integrative Neuroscience Laboratory in the Department of Psychology, Southern Illinois University, Carbondale, as an Assistant Researcher on EEG data processing. Her research interests include biomedical signalprocessing, image processing and statistical pattern classification. 


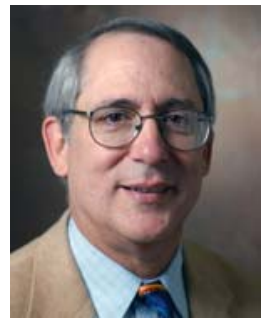

D. L. Molfese. B.A., Psychology, The Oklahoma City University, Oklahoma City , OK, 1969, M.S., Psychology, The Pennsylvania State University, State College, PA, 1970, Ph.D., Psychology, The Pennsylvania State University, State College, PA, 1972. Major field of study: developmental neuroscience.

He is currently Distinguished University Scholar and Professor in the Birth Defects Center at the University of Louisville, Louisville, KY. Previously, he served as Chair of the Department of Psychological and Brian Sciences at the University of Louisville (1999-2006), Chair and Professor of Behavioral \& Social Sciences in the School of Medicine at Southern Illinois University (1992-1999), and Professor of Psychology at Southern Illinois University (1972-1999). He is the author of 150+ journal articles and books. His current research interests include predicting cognitive and linguistic skills from infancy; developmental changes in brain, language and cognitive processes from infancy; impact of space travel on cognition, brain function and sleep; development of intervention strategies for learning disabilities in infancy, man - machine interactions using neuroelectrophysiological techniques. Sample publications include: Molfese, D., Molfese, V., Barnes, M., Warren, C., \& Molfese, P. (2008). Familial predictors of dyslexia: Evidence from preschool children with and without familial dyslexia risk. In V. Berninger, A. Fawcett, G. Reid \& L Siegel (Eds). Handbook of Dyslexia. New York Sage Publications, 99-120; Tan, A. \& Molfese, D. L. (2008). ERP Correlates of Noun and Verb Processing in Preschool-Age Children. Biological Psychology.

Dr. Molfese is a Fellow of the American Psychological Association and the American Psychological Society, as well as an elected member of The Belgian Society of Electromyography and Clinical Neurophysiology, Phi Kappa Phi, Psychonomic Society, Sigma Xi. He is also a member of the American Academy of Sleep Medicine, the Society for Neuroscience, the Acoustical Society of America, the International Neuropsychological Society, and the Society for Research in Child Development. He has been recognized as Psychologist of the Year in 2006 by the Kentucky Psychological Association, Collaborative Investigator to the National Institute of Child Health and Human Development in 2005, received the Dean's Award for 2004-2005 Outstanding Department Chair in the College of Arts \& Sciences at University of Louisville, the Phi Kappa Phi Outstanding Scholar Award in 1997, and the University Outstanding Scholar Award in 1990 from Southern Illinois University. He also received the Sigma Xi Kaplan Research Award for Significant Contributions in Child Psychology in April, 1987. 


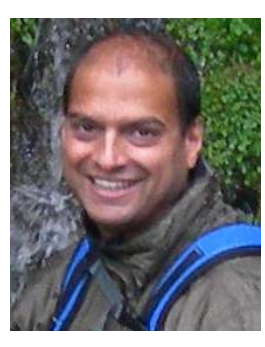

Ravi Vaidyanathan received the M.S. and Ph.D. degrees in mechanical engineering from Case Western Reserve University, Cleveland, OH, USA in 1996 and 2001 respectively.

He is presently a Lecturer in Biodynamics at the Bristol Robotics Laboratory in the University of Bristol, Bristol, UK with a co-appointment as an Assistant Professor in Systems Engineering at the US Naval Postgraduate School, Monterey, CA, USA. His research interests include biologically inspired robotics, human-machine interface, and complex adaptive systems. He has served as Principal Investigator in more than 20 research separate programmes in the US, Singapore, and UK, has authored over 50 refereed publications, and two patents.

Dr. Vaidyanathan has been the recipient of several awards from the IEEERobotics and Automation Society, the Robotics Society of Japan (RSJ), and the American Institute of Aeronautics and Astronautics (AIAA), including Best Paper in Conference (2004) at the IEEE International Conference on Intelligent Robots and Systems (IROS) and being a finalist for the New Technology Foundation (NTF) Award on Entertainment and Robotic Systems celebrating top innovations in robotics from 1987-2007. 